\title{
Paraplegia
}

\section{Post-traumatic Spinal Cord Cysts Evaluated by Magnetic Resonance Imaging}

\author{
H. A. Backe, MD,${ }^{1}$ R. R. Betz, MD, ${ }^{1}$ M. Mesgarzadeh, $M D,{ }^{2}$ T. Beck, RN, \\ M. Clancy, $\mathbf{M D}^{1}$ \\ ${ }^{1}$ Shriners Hospitals for Crippled Children, 8400 Rossevelt Boulevard, Philadelphia \\ Unit, Philadelphia, Pennsylvania 19152, ${ }^{2}$ Temple University Hospital, 3401 North \\ Broad Street, Philadelphia, PA 19140, USA.
}

\section{Summary}

In order to determine a more accurate prevalence of post-traumatic spinal cord cysts (PTSCC) in spinal cord injured (SCI) patients, we retrospectively reviewed magnetic resonance scans from symptomatic imaging and asymptomatic SCI patients. We found the incidence of PTSCC to be $51 \%$ in our patient population. The only symptom that correlated to the presence of a cyst was spasticity. The cyst develops at the site of injury and appears to be a common sequela of SCI. We believe that conservative treatment is indicated in most patients with a PTSCC.

Key words: Post-traumatic spinal cord cyst; Syringomyelia; Paraplegia; Magnetic resonance imaging.

Post-traumatic spinal cord cysts (PTSCC), or syringomyelia, are cystic lesions which are thought to be a rare sequela of spinal cord injury (SCI), occurring in less than $3 \cdot 2 \%$ of SCI patients (Rossier, 1985). Late deterioration and worsening of neurological function in SCI patients has been attributed to the enlargement of these cysts (Rossier et al., 1985; Barnett and Jousse, 1973; Eismont et al., 1984; Quencer et al., 1983; Dworkin and Staas, 1985; Rossier et al., 1968; Jensen and Reske-Nielsen, 1977). Until the advent of magnetic resonance imaging (MRI), the diagnosis of PTSCC was made using CT myelography.

PTSCC's are thought to develop from regions of haemorrhage or ischaemia within the spinal cord. Rossier (1985) believed the dorsal columns to be the most susceptible. Haematomyelia breaks down, forming cystic regions which contain a high-protein content relative to normal cerebrospinal fluid. In myelomalacia or ischaemic regions of injury, necrobiosis occurs and results in cystic cavities in the cord. These cysts may communicate with the fourth ventricle, central canal, or subarachnoid space via Virchow-Robin spaces. 
Enlargement of a cyst is thought to occur secondary to increases in pressure gradients between different regions of the cord, resulting in upward expansion (Barnett and Jousse, 1973; Eismont et al., 1984). Barnett and Jousse suggested that glial cells lined the cysts and secreted an exudate, causing expansion.

The most commonly reported signs and symptoms on presentation are back pain, hyperaesthesia, dysreflexia, and spasticity. Rossier's patients also presented with ascending sensory levels, depressed tendon reflexes, and increased motor deficits (1985). In these symptomatic patients the diagnosis was confirmed by CT myelography, and he determined his prevelence for PTSCC from this series of patients. It has been suggested that the prevalence of PTSCC may be higher; that is, present in asymptomatic patients as well (Eismont et al., 1984; Quencer et al., 1983). If this is so, the true prevalence of PTSCC cannot be determined in series consisting of only symptomatic patients. With MRI, detection of a cyst and differentiation from myelomalacia has become feasible, more specific, and noninvasive.

The purpose of this paper was to retrospectively review the routine MRI scans of patients with SCI admitted to our institution for rehabilitation. We then calculated the prevalence of PTSCC in our population, including both symptomatic and asymptomatic patients, and cross-analysed PTSCC with the most common signs and symptoms on presentation.

\section{Methods}

We reviewed the records of 96 acute SCI patients who were admitted to the Philadelphia Unit for rehabilitation between 1984 and 1989. Of these, 88 patients underwent routine MRI scans of the spine on admission as a baseline study. Of the 88 scans, there was adequate resolution on the sagittal view in 63 scans to determine the presence or absence of a cyst. Each of these scans was read jointly by two of the authors. The scans contained both T1 and T2 images of the spine and cord in the region of injury. In some patients the entire cord was imaged. A welldefined area of low-signal intensity within the spinal cord seen on a T1 weighted image was determined to be 'definitely a cyst' (Fig. 1). In some cases, the lesions

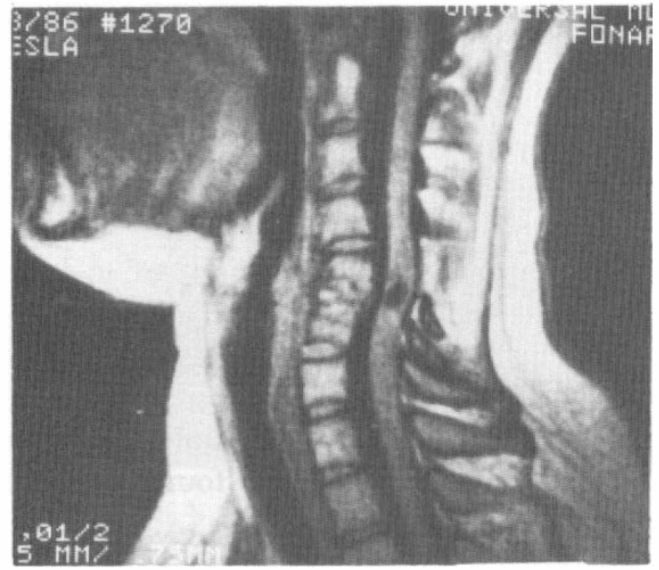

Figure 1 Sagittal MRI of cervical spine (T1 weighted image) demonstrating clearly delineated area of low signal intensity (cyst) within the spinal cord at the area of bony injury. 
were not typical of a cyst due to poor resolution, the size of the cyst, and/or decreased signal intensity. These were categorised as 'probably a cyst' or 'probably not a cyst,' determined by the radiologist (M.M.) according to the degree of signal intensity and size of the cyst. In other cases, the cord was normal, or there was no lesion present, and these were categorised as 'no cyst.' The date of study, length of time post-injury, presence or absence of a cyst (definitely, probably, probably not, and none), location (highest level of cyst involvement), and area (in two dimensions on the sagittal view measured in $\mathrm{cm}^{2}$ ) were recorded.

Independently, without knowledge of the MRI, one investigator recorded the age, date and level of injury, Frankel classification, sensory and motor levels, total motor index, and presence or absence of spasticity, dysreflexia, back pain, and hyperaesthesia. Spasticity and hyperaesthesia were categorised as absent, moderate, or severe.

Both sets of data were combined and analysed statistically using two-way and multi-way frequency tables and BMDP statistical software. The presence, location, and size of a cyst as seen on T1 weighted images were correlated to the number of days post-injury, age of the patient, injury level, presenting symptoms, and Frankel classification. The prevalence of PTSCC in our population was also calculated.

\section{Results}

The mean age of patients studied was $15 \cdot 6$ years (range $2-22$ years). The mean time interval post-injury was 1.6 years (range 2 days-20 years). The presence of PTSCC in our population was 32 of 63 , or $51 \%$, over 15 times higher than previously reported (Table I). Cyst location always correlated to the level of injury $(\mathbf{P}<0.001)$ (Table II). Since greater than $50 \%$ of the injuries in this series were located in the

Table I Presence of cyst

\begin{tabular}{lrccrc}
\hline Age (Yrs) & No & $\begin{array}{c}\text { Probably } \\
\text { No }\end{array}$ & $\begin{array}{c}\text { Probably } \\
\text { Yes }\end{array}$ & Yes & Total \\
\hline 13 or under & 6 & 2 & 0 & 9 & 17 \\
14 to 18 & 7 & 2 & 2 & 11 & 22 \\
Over 18 & 13 & 1 & 3 & 7 & 24 \\
Total & 26 & 5 & 5 & 27 & 63 \\
\hline
\end{tabular}

Table II Location of cyst

\begin{tabular}{lcccccc}
\hline $\begin{array}{l}\text { Level of } \\
\text { injury }\end{array}$ & $\begin{array}{c}\text { Total number of } \\
\text { injuries examined }\end{array}$ & C1-C8 & T1-T8 & T9-T12 & L1-S & Total \\
Cervical & 39 & 19 & 1 & 0 & 0 & 20 \\
High thoracic & 13 & 0 & 3 & 0 & 0 & 3 \\
Low thoracic & 5 & 0 & 0 & 5 & 0 & 5 \\
Lumber & 1 & 0 & 0 & 0 & 1 & 1 \\
Total & 63 & 19 & 4 & 5 & 1 & $29^{*}$ \\
\hline
\end{tabular}

*3 patients excluded due to incomplete data. 
cervical spine and were typed Frankel A, it was expected that there would be strong correlations between sensory and motor levels and level of injury, and there were $(p=0.0011$ and $p=0.001$, respectively).

Although the majority of patients had cervical spine injuries, no particular level of the cord appeared more susceptible to cyst development than other levels.

A relationship was found between patient complaints of spasticity and the presence of a cyst. The majority of patients without spasticity did not have a cyst, while those who had severe spasticity (that is, presence of spasticity severe enough to significantly interfere with activities of daily living) had evidence of a cyst $(\mathbf{P}=$ 0.02 ) (Table III). The size of the cyst was evaluated with regard to spasticity, and no correlation was noted (Fig. 2).

The presence of dysreflexia, back pain, or hyperaesthesia did not correlate with the presence or size of a cyst.

\section{Discussion}

The use of MRI to evaluate SCI now enables physicians to easily diagnose PTSCC. MRI has been shown to be more specific than delayed CT myelography in evaluating myelomalacia and cysts (Bradway, 1986; Betz, 1987). In this study, as in

Table III Prevalence of cyst vs. severity of spasticity

\begin{tabular}{lccccc}
\hline Spasticity & No & $\begin{array}{c}\text { Prevalence of Cyst } \\
\text { Probably } \\
\text { No }\end{array}$ & $\begin{array}{c}\text { Probably } \\
\text { Yes }\end{array}$ & Yes & Total \\
\hline Absent & 18 & 2 & 1 & 9 & 30 \\
Moderate & 5 & 3 & 1 & 7 & 16 \\
Severe & 3 & 0 & 3 & 11 & 17 \\
Total & 26 & 5 & 5 & 27 & 63 \\
\hline
\end{tabular}

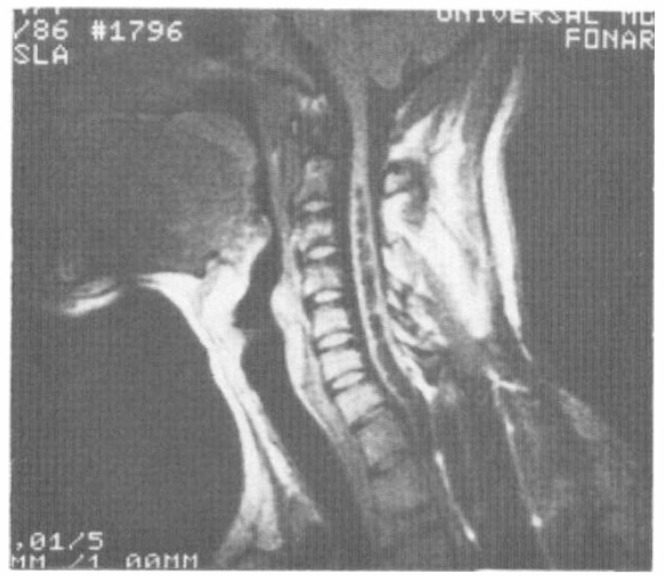

Figure 2 Sagittal MRI of cervical spine (T1 weighted image). Large cyst seen in a quadriplegic patient with minimal complaints of spasticity. 
a previous study by one of us (Betz, 1987), we found that sagittal T1 weighted images were most helpful in evaluating the spinal cord for a cyst.

Unlike previous investigators, who included only symptomatic patients, we calculated the prevalence of PTSCC in all SCI patients in our series, both symptomatic and asymptomatic. Our prevalence was 51\%, much higher than previously reported. None of the patients in our series presented with ascending myelopathy; therefore, we are unable to compare the prevalence in our patients with that of other investigators. Our data suggests that PTSCCs are common sequelae of SCI. They are probably stable in the majority of patients but in rare instances can enlarge to cause increasing motor weakness and sensory loss.

We found a high correlation between the location of the cyst and the initial level of cord injury (Table II). This was expected, since uninjured areas of the cord should not develop haematomyelia or myelomalacia.

Some cysts extended to regions above the injured segement of the cord. This could have been caused by upward expansion of the cyst from pressure gradients, or from the initial zone of cord injury extending proximally. However, none of the patients in our series had evidence of ascending neurological loss.

Rossier (1985) found a higher prevalence of PTSCC in tetraplegic patients when compared to paraplegic patients $(4 \cdot 5 \%$ vs. $1 \cdot 7 \%)$, but the difference was insignificant. We found similar prevalences for both tetraplegics (50\%) and paraplegics $(48 \%)$, and similar prevalences were found for those patients with an ASIA total motor index $<50$ or $\geq 50$. We therefore believe there is no correlation between the severity of injury and the incidence of PTSCC.

Spasticity was the only symptom which showed any valid correlation to the presence of a cyst $(\mathrm{p}=0.02)$ (Table III). However, our data lacks large numbers of patients with severe spasticity. We therefore hesitate to state a definite relationship between presence of PTSCC and spasticity. Back pain, dysreflexia, and hyperaesthesia did not correlate with the presence of a cyst.

Five patients had follow-up MRI scans 2 weeks to 11 months following initial MRI. Two patients showed no change in cyst size from initial MRI, and their neurological examination findings were unchanged. One patient showed development of a cyst as early as 2 weeks post-injury, demonstrating that PTSCC may develop in the injured spinal cord soon after injury as well as later. The other two scans were of poor quality and could not be read.

The surgical management of PTSCC has consisted of draining and shunting the cystic areas into the subarachnoid space. Quencer et al., (1983) advocated shunting cysts which were symptomatic as well as large, asymptomatic cysts. We agree with Barnett (1973) that conservative treatment is acceptable if the disability is tolerable. In our series, the neurological condition did not change in any patient regardless of the size of the cyst.

From our data, spasticity was the only symptom which correlated to a cyst. This was the one symptom in Quencer's series which did not improve after shunting. Since none of our patients underwent shunting procedures, we cannot comment on its efficacy in patients with advancing neuropathy.

\section{Conclusions}

Prevalence of PTSCC is much higher than previously thought and is a common 
sequelae of SCI. We found a prevalence of $51 \%$ in our SCI population. Most patients were either asymptomatic or they tolerated their symptoms, and none presented with ascending myelopathy. The cyst always developed at the site of injury. There was no correlation between cyst size and the presence of back pain, hyperaesthesia, or dysreflexia; however, a trend was noted between the presence of a cyst and spasticity.

\section{Acknowledgements}

The authors wish to acknowledge Carolyn Hill for assistance with manuscript preparation.

\section{References}

BARNETT HJM, JouSSE AT 1973 Syringomyelia as a late sequel to traumatic paraplegia and quadriplegia-Clinical features. In: MajorProblemsinNeurology, Syringomyelia, Vol. I. Barnett HJM, Foster JB, Hudgson P, eds. W.B. Saunders, London, pp. 129-153.

BetzRR,Gelman AJ, DeFilippGJ, Mesgarzadeh M, Clancy M, SteelHH 1987 Magnetic resonance imaging in the evaluation of spinal cord injured children had adolescents. Paraplegia 25:92-99.

BRADWAY JK, KAYANAGH BF, HoUSER OW 1986 Post-traumatic spinal-cord cyst. A case report. F Bone foint Surg 68A(6):923-933.

DWORKIN GE, StAas WE JR 1985 Post traumatic syringomyelia. Arch Phys Med Rehab 66:329-331.

EISMONT FJ, GREEN BA, QUENCER RM 1984 Post-traumatic spinal cord cyst. A casereport. F Bone Foint Surg 66A(4):614-618.

JENSEN F, RESKe-NIELSEN E 1977 Post-traumatic syringomyelia: Review of literature and two new autopsy cases. Scanf Rehab Med 9:35-43.

QUENCERRM, GREEN BA, EISMONT FJ 1983 Post-traumatic spinal cord cysts: clinical features and characterization with metrizamide computer tomography. Radiology 146:415-423.

ROSSIER AB, WERNER A, WILDIE, BERNEY J 1968Contributions to the study of late cervical syringomyelic syndromes after dorsal or lumbar traumatic paraplegia. $\mathcal{F}$ Neurol Neurosurg Psychiatry 31:99-105.

Rossier AB, Foo D, Shillito J, Dyro FM 1985: Post traumaticcervical syringomyelia. Incidence, clinical presentation, electrophysiological studies, syrinx protein, and results of conservativeand operative treatment. Brain 108:439-461. 